\title{
Control System Research of a New Hybrid Magnetic Levitation Motor
}

\author{
Li Zeng ${ }^{\text {a, }}$, Zhida Zhu ${ }^{\text {a }}$, Fan Zhang ${ }^{\text {a }}$, Fang Chen ${ }^{\text {a }}$, Jin Sun ${ }^{\text {a }}$ \\ ${ }^{a}$ College of Mechanical Engineering Yangzhou University, Yangzhou 225127, China \\ *Corresponding Author: lizengcf@163.com
}

\begin{abstract}
A new control structure scheme of magnetic levitation motor is presented. Its working principle is introduced, and the mathematical model of the magnetic levitation force is established based on principle of virtual displacement. The distribution characteristics of electromagnetic levitation force and magnetic induction intensity of the motor are analyzed with the finite element method.
\end{abstract}

Keywords: magnetic levitation motor, working principle, mathematical model, finite element analysis.

\section{Introduction}

Magnetic levitation motor is one of the latest technologies of the motor which use magnetic levitation force to do the work of traditional bearings. The old magnetic levitation motor usually uses two sets of threephase windings, one set of windings is used to produce rotating magnetic field, the other set of windings work with the driving one to produce levitation force. This kind of magnetic levitation motor needs occupy large structure space. On the other side, the magnetic levitation motor with a single set of windings is more reasonable, and occupies smaller space. Therefore a AC magnetic levitation motor is proposed, which has a stator with three section structure windings and a rotor using permanent magnet.

\section{The Principle of the New AC Magnetic Levitation Motor}

The stator of the new AC magnetic levitation motor has 3 independent controlled windings: a, b, c. and the motor work with three-phase alternating current: A, B, C. As shown in Figure 1, the phase windings a is composed of A and $\mathrm{C}$ phase of three-phase alternating current windings. As the phase windings a, the phase windings $b$ is composed of
B and A phase of three-phase alternating current windings, and the phase windings $\mathrm{c}$ is composed of $\mathrm{C}$ and $\mathrm{B}$ phase of three-phase alternating current windings. These three phase windings are connected in a special order, and then they would produce the three-phase rotating magnetic fields which are shown in Figure 2. Because of the symmetry of these rotating magnetic fields, the resultant force of magnetic pulling force, which produced by these rotating magnetic fields, is 0 .

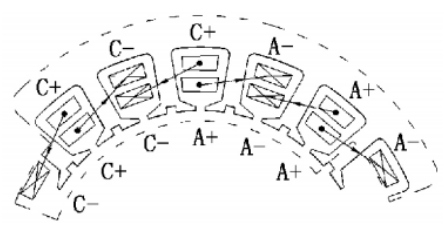

Fig. 1 the phase windings a of AC magnetic levitation motor

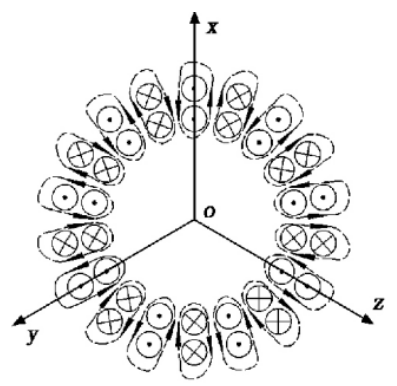

Fig. 2 the rotating magnetic fields formed by AC magnetic levitation motor

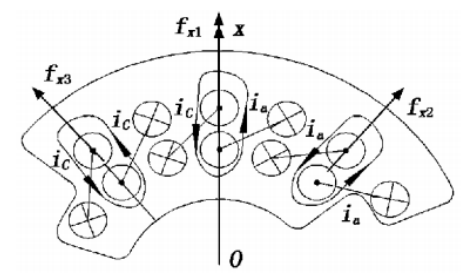

Fig. 3 the magnetic pulling force produced by the phase windings a

According to the study of the phase windings a, when the lines $i_{a}$ and $i_{c}$ in the phase windings a has the equal size current $\Delta i_{x}$, they would produce the distribution of magnetic pulling force shown as Figure 3. According to the 
symmetric distribution of the increased magnetic field about $\mathrm{X}$-axial, the resultant force must follow in the $\mathrm{X}$ direction. In the same way, the magnetic levitation force of produced by the phase windings $\mathrm{b}$ or $\mathrm{c}$ is also along the $\mathrm{Y}$ axis or the $\mathrm{Z}$ axis. So the control of current increment $\Delta i_{x}$, $\Delta i_{y}$ and $\Delta i_{z}$ can keep the rotor at the center of the balance sections.

\section{The Levitation Force Model of the of the New AC Magnetic Levitation Motor}

To neglect the magnetization effect of the iron core, the magnetic pulling force of a single magnetic circuit of the stator windings is analyzed as shown in Figure 4.

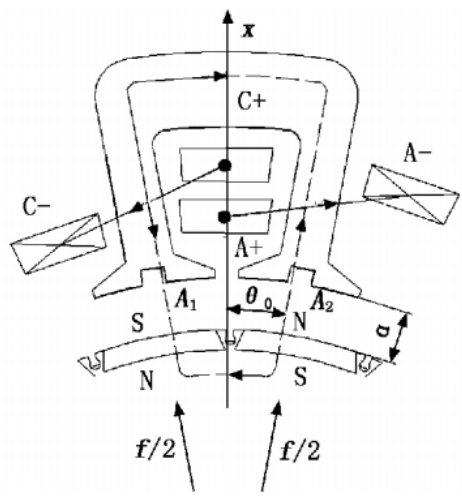

Fig. 4 the magnetic pulling force of a single magnetic circuit

The area of the core shoe is $A_{1}=A_{2}=A_{a}$. The distance between the core shoe and the rotor is $a . B_{a}$ is the magnetic flux density between the core shoe and the rotor. $H_{a}$ is the magnetic field intensity between the core shoe and the rotor. $\theta_{0}$ is the angle between the center line of the core shoe and the $\mathrm{X}$ axis. Then the energy storage between the two core shoes is as follows:

$$
w=0.5 B_{a} H_{a} V_{a}=0.5 B_{a} H_{a} A_{a}(2 a)=\frac{a \cdot A_{a} \cdot B_{a}^{2}}{\mu_{0} \bullet \mu_{r}}
$$

Where $V_{a}$ is the volume of the core shoe. $\mu_{0}$ is permeability of vacuum. $\mu_{r}$ is the relative permeability of the stator. For the small displacement $d a, A_{a}$ and $B_{a}$ are usually unchanged. So the formula 2 is derived from the virtual displacement principle.

$$
f=\frac{d w}{d a}=\frac{B_{a}^{2} \cdot A_{a}}{\mu_{0} \bullet \mu_{r}}
$$

Where $f$ is the magnetic levitation pulling force, which is perpendicular to the rotor surface and along the direction of the magnetic field. Therefore

$$
f_{x 1}=f \cdot \cos \theta_{0}=\frac{B_{a}^{2} \cdot A_{a} \cdot \cos \theta_{0}}{\mu_{0} \bullet \mu_{r}}
$$

There are 3 effective magnetic paths in the phase windings a (as shown in Figure 3). In combination with formula 3 , the following formula 4 is gotten.

$$
f_{x}=f_{x 1}+f_{x 2} \cos 40^{\circ}+f_{x 3} \cos 40^{\circ}=f_{x 1}\left(1+2 \cos 40^{\circ}\right)
$$

$B_{a}$ is the resultant magnetic flux density at the air gap between the core shoe and the rotor, which is composed of the magnetic flux density $B_{p m}$ provided by the permanent magnet in rotor and the magnetic flux density $B_{c}$ provided by the coil. That is $B_{a}=B_{p m}+B_{c}$.

The calculation process of $B_{p m}$ and $B_{c}$ is as following.

(1) $B_{p m}$ can be regarded as the magnetic flux density of permanent magnet, while the motor works without load ${ }^{[1]}$. As shown in Figure 5, the magnetic flux density of a fan-shaped permanent magnet can be equivalent to the magnetic flux density produced by the coils surrounding the permanent magnet with infinite power, the magnetic flux density is generated by the around equivalent line current at where it is $r=R_{2}$.

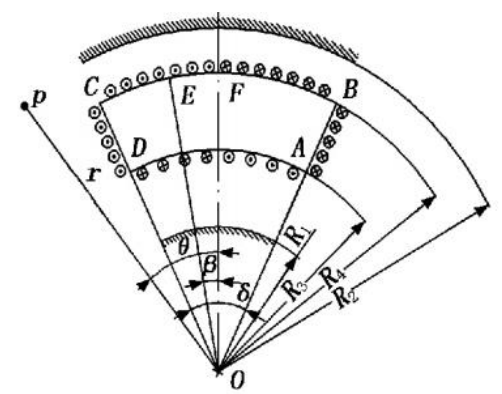

Fig. 5 the equivalent magnetic field of permanent magnet

For the sake of convenience in the computation, the average magnetic flux density in the $\delta$ angle of the permanent magnet is finally used for the calculation of the magnetic pulling force. The magnetic flux density calculation is solved by the Laplasse equation of the magnetic scalar $\varphi$ in the polar coordinates.

$$
\frac{\partial^{2} \varphi}{\partial r^{2}}+\frac{1}{r} \cdot \frac{\partial \varphi}{\partial r}+\frac{1}{r^{2}} \cdot \frac{\partial \varphi}{\partial \theta^{2}}=0
$$

The formula 5 is a differential calculation of the magnetic scalar $\varphi$ which is distributed in the area with a radius of $r$ between the center line $O F$ and point $p$ which unfold along counter clockwise direction from $O F$ to angle $\theta$. The magnetic permeability at $R_{2}$ and $R_{1}$ is infinitely large, so the tangential component of magnetic flux density at $R_{2}$ and $R_{1}$ is 0 .

Because the magnetic flux density $B=\mu_{0} H=-\mu_{0} \operatorname{grad} \varphi$, the magnetic flux density of a single coil at $R_{2}$ can be calculated. Then from and then the magnetic flux density produced by $A B$ and $C D, A D, B C$ at $R_{2}$ is also obtained. 


$$
\begin{aligned}
& B_{p m}=B_{A B}-B_{B C}-B_{D A}=\sum_{m=0}^{\infty} F_{a n R_{1}} \cos (n \theta)+ \\
& \sum_{m=0}^{\infty} F_{a n R_{2}} \cos (n \theta)+\sum_{m=0}^{\infty} F_{a n R_{3}} \cos (n \theta) \\
& =\sum_{m=0}^{\infty}\left(F_{a n R_{1}}+F_{a n R_{2}}+F_{a n R_{3}}\right) \cos (n \theta) \\
& \text { And } \quad F_{a n R 1}=F_{n 1} \sin \left(\frac{n \delta}{2}\right) \cdot\left(\frac{R_{4}^{n+1}-R_{3}^{n+1}}{n+1}+R_{1}^{2 n} \cdot F_{n 2}\right) \\
& F_{n 1}=2 P \cdot \frac{2 \mu_{0}}{\pi} \cdot \frac{k_{m} \cdot B_{r}}{\mu_{0}} \cdot \frac{R_{2}^{n-1}}{R_{2}^{2 n}-R_{1}^{2 n}} \\
& F_{n 2}= \begin{cases}\ln \left(\frac{R_{4}}{R_{3}}\right) & n=1 \\
\frac{R_{4}^{n+1}-R_{3}^{n+1}}{n+1} & n \neq 1\end{cases} \\
& F_{a n R_{2}}=-0.5 F_{n 1} F_{n 3} \frac{R_{3}^{2 n}+R_{1}^{2 n}}{R_{3}^{n-1}} \\
& F_{n 2}= \begin{cases}\frac{R_{2}}{2}-\frac{\sin \delta}{2} & n=1 \\
\frac{\sin [(n-1) \delta / 2]}{n-1}-\frac{\sin [(n+1) \delta / 2]}{n+1} & n \neq 1\end{cases} \\
& F_{a n R_{3}}=-0.5 F_{n 3} \frac{R_{4}^{2 n}-R_{1}^{2 n}}{R_{4}^{n-1}}
\end{aligned}
$$

Where $P$ is the pair number of stator pole. $0^{\circ} \leq \theta \leq 360^{\circ}$. $k_{m}=\frac{1+0.75 x_{m}}{1+x_{m}}, x_{m}$ is the magnetic susceptibility of magnet. $B_{r}=-\mu_{0} \bullet \frac{\partial \varphi}{\partial r} ; n=(2 m+1) \cdot P, m=0,1,2,3 \ldots$.

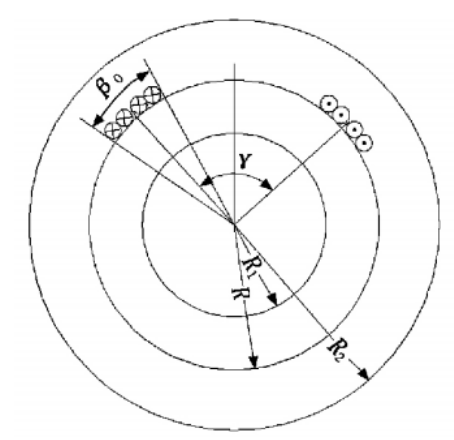

Fig. 6 the equivalent distribution of a set of windings in the stator slot

(2) $B_{c}$ is the magnetic field intensity provided by the coil at $R_{2}$, and the coil inside the slot is equivalent to the surface current layer evenly which is distributed in the range of slot $\beta_{0}$ and is attached to the surface of the stator iron core ${ }^{[2]}$. That is shown in Figure 6. The coil can be regarded as an ideal coil which is evenly distributed in the range of $\beta_{0}$. The center line of ideal coil coincides with the actual central line. The actual cross angle of the coil is $\gamma$. The ideal current element is $d i=\frac{N I}{\beta_{0}} d \theta \quad(N$ is the number of coil turns, $I$ is the current of coil). The radius of the coil is $R=R_{2}$.

The calculation process of the magnetic field intensity produced by the coil is similar to the one of the permanent magnet. So the following formula is finally gotten.

$$
B_{c}=\sum_{n=1}^{\infty} \frac{1}{n_{0}} \cdot F_{n 6} \cdot \frac{N I}{\beta_{0}} \cdot \sin \left(\frac{n_{0} \gamma}{2}\right) \cdot \sin \left(\frac{n_{0} \beta_{0}}{2}\right) \cdot \cos \left(n_{0} \theta\right)
$$

And $F_{n 6}=2 P_{0} \cdot \frac{2 \mu_{0}}{\pi} \cdot \frac{1}{R_{2}} \cdot \frac{R_{2}^{2 n}+R_{1}^{2 n}}{R_{2}^{2 n}-R_{1}^{2 n}}$.

Where $P_{0}$ is the the number of stator coil turns. $n_{0}=1,2$, 3....

\section{Finite Element Analysis of Levitation Force of the New AC Magnetic Levitation Motor}

The parameters of AC magnetic levitation motor modeling are shown in Table 1. The finite element analysis model is established with the parameters of motor and the structure mesh map of motor motion boundary is shown in Figure 7.

Table 1 Parameters of AC Magnetic Levitation Motor

\begin{tabular}{|c|c|}
\hline Parameter & Value \\
\hline external diameter of stator $(\mathrm{mm})$ & 165 \\
\hline internal diameter of stator $(\mathrm{mm})$ & 105 \\
\hline tooth width of stator $(\mathrm{mm})$ & 15.30 \\
\hline air gap width $(\mathrm{mm})$ & 0.75 \\
\hline axial length $(\mathrm{mm})$ & 14 \\
\hline the number of coil turns & 170 \\
\hline
\end{tabular}

Fig. 7 the structure mesh map of motor motion boundary

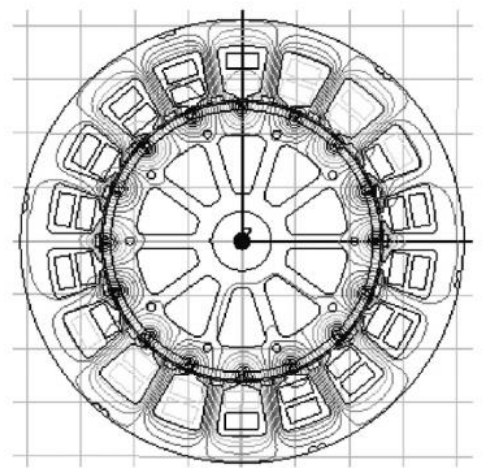

Fig. 8 distribution of magnetic flux lines without load

Figure 8 is the distribution of the magnetic flux line when the motor works without load. Figure 9 is the curve of 
radial magnetic flux value at the air gap of the motor when it works without load. Figure 10 is the curve of radial magnetic flux value at the air gap of the motor after phase windings a has loaded. According to Figure 8 and Figure 9, we can see that the distribution interval of magnetic flux peak is $20^{\circ}$. According to figure 9 and figure 10, we can see that the magnetic flux of air gap at the core shoe is obviously increased, and the magnetic flux increase of air gap on the two sides of vertical pole is the most obvious. This conforms to the hypothesis proposed before.

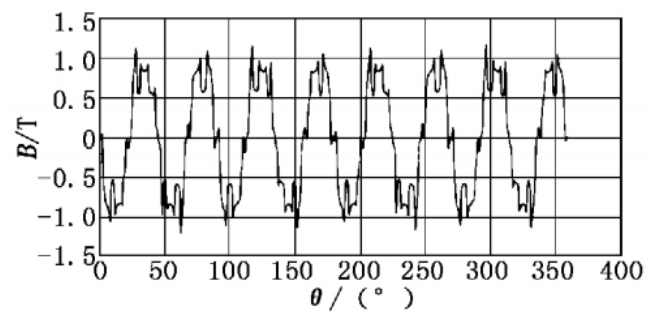

Fig. 9 the curve of radial magnetic flux value at the air gap of the motor without load

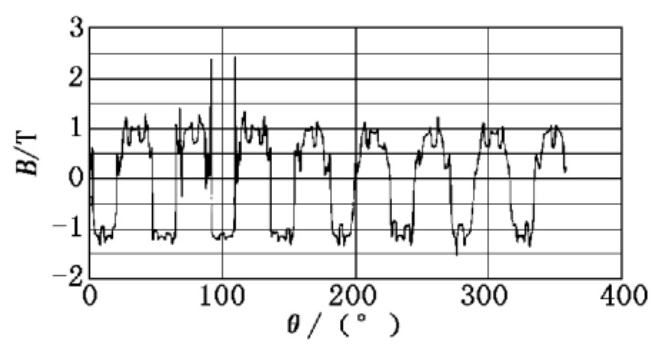

Fig. 10 the curve of radial magnetic flux value at the air gap of the motor after phase windings a has loaded

When a set of magnetic levitation winding works in the motor, the rotor magnetic pulling force in $\mathrm{Y}$ direction would be noted down, while the displacement of rotor in $\mathrm{Y}$ direction is changed. The relationship of the magnetic levitation force and the radial displacement of rotor is shown in Figure 11.

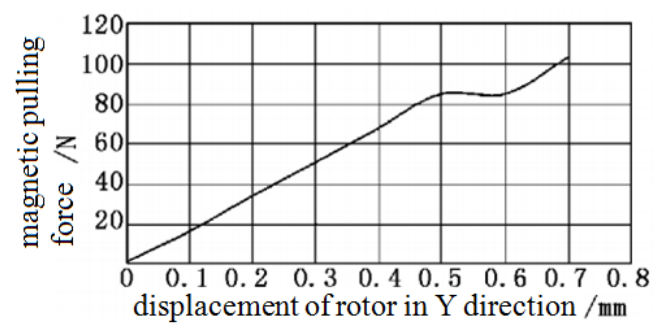

Fig. 11 unidirectional magnetic levitation force

From Figure 11, we can see that the magnetic pulling force increases gradually, while the deviation of the rotor from the center position gets bigger (the air gap in the $\mathrm{Y}$ positive direction is reduced). and the curve has a good linearity in the small displacement range.

\section{Conclusions}

a new three section structure windings control structure of magnetic levitation motor is presented, which can solve the problem of more space occupied by the excessive windings very well in the magnetic levitation motor. With the help of the finite element analysis, the feasibility of this structure can be obtained. The optimization of this structure needs the further studies in the future.

\section{Acknowledgment}

Thank the China Natural Science Foundation for this project, Item Number: 51375427, 51475409. Thank the Jiang Su Natural Science Foundation for this project, Item Number: BY2014117-08, BY2015061-04. Thank the Yangzhou city - Yangzhou University of Science and Technology Cooperation Program Funds, Item Number: SXT20140041, YZ2016244, YZ2016255. Thank the Research Project of State Key Laboratory of Mechanical System and Vibration, Item Number: MSV201810.

\section{References}

(1) Boules N: "Prediction of no-load flux density distribution in PM machines", IEEE Trans On Industrial Application, Vol. 21, No. 4, pp. 633-643, 1985

(2) Chen Yangsheng, Lin Youyang: "Analysis and calculation of air gap magnetic density of permanent magnet motor", China Journal of electrical engineering, Vol. 14, No. 5, pp. 18-26, 1994.

(3) Deng Zhiquan and Yan Yangguang: "Basic theory and current study situation of no bearing ac motor", Transactions of China Electrotechnical Society, 2000,15(2):30-35.

(4) Xu Kaiguo : "Study and design of rotor of permanent magnetic levitation wind turbines", Wuhan University of Technology Press, 2008:14-16.

(5) Tan Qingchang, Liu Mingjie and Meng Huiqin : "Calculation of carrying capacity and stiffness of permanent magnet centripetal bearing", Journal of Tribology, 1994,14(4):337-344.

(6) Xie Longhan, Geng Yu and Qiu Wan : “ANSYS analysis of electromagnetic field", Electronic Industry Press, 2012.

(7) Hu Yiefa, Zhou Zude, and Jiang Zhengfeng : "Basic theory and application of magnetic bearing", China Machine Press, 2006. 